JOURNAL OF THE

AMERICAN MATHEMATICAL SOCIETY

Volume 11, Number 4, October 1998, Pages 751-770

S 0894-0347(98)00274-4

\title{
FACTORIZATION AND APPROXIMATION PROBLEMS FOR MATRIX FUNCTIONS
}

\author{
V. V. PELLER
}

\section{INTRODUCTION}

The purpose of this paper is to develop a new approach to certain approximation and factorization problems for matrix-valued functions. The approach is based on studying properties of maximizing vectors of Hankel operators with matrix-valued symbols and on the solution of the so-called recovery problem for unitary-valued matrix functions. In the case of scalar functions such problems were studied in detail in $[\mathrm{PK}]$. It turns out however, that the case of matrix functions is considerably more complicated than the scalar case.

In this paper we solve the recovery problem with the help of Hankel operators. This solution also allows us to give a new approach to Wiener-Hopf factorization problems and obtain some new results. Another application we consider here is the problem of finding a superoptimal analytic approximation to a matrix function on the unit circle. We improve earlier results on hereditary properties of such approximations (see [PY1]) and on continuity properties of the operator of superoptimal approximation (see [PY3]).

Recall that for a bounded $m \times n$ matrix-function $\Phi$ on the unit circle $\mathbb{T}$ the Hankel operator $H_{\Phi}$ is defined on the Hardy class $H^{2}\left(\mathbb{C}^{n}\right)$ of $\mathbb{C}^{n}$-valued functions by

$$
H_{\Phi} f=\mathbb{P}_{-} \Phi f, \quad f \in H^{2}\left(\mathbb{C}^{n}\right),
$$

where $\mathbb{P}_{-}$is the orthogonal projection from $L^{2}\left(\mathbb{C}^{m}\right)$ onto $H_{-}^{2}\left(\mathbb{C}^{m}\right) \stackrel{\text { def }}{=} L^{2}\left(\mathbb{C}^{m}\right) \ominus$ $H^{2}\left(\mathbb{C}^{m}\right)$.

The Toeplitz operator $T_{\Phi}: H^{2}\left(\mathbb{C}^{n}\right) \rightarrow H^{2}\left(\mathbb{C}^{m}\right)$ is defined by

$$
T_{\Phi} f=\mathbb{P}_{+} \Phi f, \quad f \in H^{2}\left(\mathbb{C}^{n}\right),
$$

where $\mathbb{P}_{+}$is the orthogonal projection from $L^{2}\left(\mathbb{C}^{m}\right)$ onto $H^{2}\left(\mathbb{C}^{m}\right)$.

We denote by $\mathbb{M}_{m, n}$ the space of $m \times n$ complex matrices. If $X$ is a function space, for a matrix-valued (or a vector-valued) function $f$ we write $f \in X$ if all entries of $f$ are in $X$. In case we want to specify the size of the matrix-valued (or vector-valued) function, we write $f \in X\left(\mathbb{M}_{m, n}\right)$ (or $f \in X\left(\mathbb{C}^{n}\right)$ ).

The recovery problem for unitary-valued functions can be stated in the following way. Let $U$ be an $n \times n$ unitary-valued function. Let $X$ be a space of functions on $\mathbb{T}$. Suppose we know that $\mathbb{P}_{-} U \in X$. The problem is: for which spaces $X$ can we conclude under certain natural assumptions on $U$ that $U \in X$ ?

Received by the editors June 11, 1997.

1991 Mathematics Subject Classification. Primary 47B35, 30Dxx, 46Exx.

The author is partially supported by NSF grant DMS 9304011. 
Certainly, without any additional assumption on $U$ the condition $\mathbb{P}_{-} U \in X$ does not imply any nontrivial conclusion. For example, if $U$ is in $H^{\infty}$, then $\mathbb{P}_{-} U=\mathbb{O}$ but we cannot say anything nontrivial about $U$. The problem becomes reasonable if we assume that the Toeplitz operator $T_{U}: H^{2}\left(\mathbb{C}^{n}\right) \rightarrow H^{2}\left(\mathbb{C}^{n}\right)$ is Fredholm or if we assume that $T_{U}$ has dense range in $H^{2}\left(\mathbb{C}^{n}\right)$.

In $[\mathrm{PK}]$ the recovery problem was considered for scalar unimodular functions (i.e., for functions $u$ satisfying $|u(\zeta)|=1$ a.e. on $\mathbb{T}$ ). Three big classes of function spaces $X$ were found in $[\mathrm{PK}]$ for which the recovery problem has an affirmative solution under the above natural assumptions on $u$. See also $[\mathrm{To}]$ where other classes of function spaces were found.

The first class of function spaces found in [PK] consists of the so-called $\mathcal{R}$-spaces, i.e., function spaces which can be described in terms of rational approximation in $B M O$ (see $\S 3$ ). The second class introduced in $[\mathrm{PK}]$ was the class of Banach spaces satisfying the axioms (A1)-(A4) (see §3) which consists only of separable Banach spaces. To be able to work with nonseparable spaces a third class of function spaces was introduced in $[\mathrm{PK}]$ which consists of spaces satisfying the axioms (B1)-(B3) (see $\S 3$ ).

In $\S 7$ we explain how to solve the recovery problem for $\mathcal{R}$-spaces (implicitly this was done in $[\mathrm{Pe} 2])$. Then we give a solution of the recovery problem for function spaces satisfying (A1)-(A4). We also solve the recovery problem for function spaces satisfying (B1)-(B3) and an additional axiom (B4) (see §3).

To solve the recovery problem for spaces satisfying (A1)-(A4) or (B1)-(B4) we study in $\S 6$ properties of maximizing vectors of Hankel operators with matrix-valued symbols.

As an application of the above results we consider the heredity problem for superoptimal approximation by analytic matrix functions. It is well known and easy to see that unlike the scalar case a continuous matrix function generically has infinitely many best approximations by analytic functions. Recall that for a bounded $m \times n$ matrix function $\Phi$ on $\mathbb{T}$ a superoptimal approximation can be defined as follows. Let $\Omega_{0}$ be the set of best approximations:

$$
\Omega_{0}=\left\{Q \in H^{\infty}: Q \text { minimizes ess } \sup _{\zeta \in \mathbb{T}}\|\Phi(\zeta)-Q(\zeta)\|\right\} .
$$

Define inductively the sets $\Omega_{j}$ by

$$
\Omega_{j}=\left\{Q \in \Omega_{j-1}: Q \text { minimizes } \operatorname{ess} \sup _{\zeta \in \mathbb{T}} s_{j}(\Phi(\zeta)-Q(\zeta))\right\}
$$

(for a matrix (or an operator) $A$ the $j$ th singular value $s_{j}(A), j \geq 0$, is the distance from $A$ to the set of matrices (operators) of rank at most $\left.j, s_{0}(A) \stackrel{\text { def }}{=}\|A\|\right)$. Elements of $\Omega_{\min \{m, n\}-1}$ are called superoptimal approximations of $\Phi$ by analytic functions (or superoptimal solutions of Nehari's problem). Put

$$
t_{j} \stackrel{\text { def }}{=} \operatorname{ess} \sup _{\zeta \in \mathbb{T}} s_{j}(\Phi(\zeta)-Q(\zeta)), \quad Q \in \Omega_{j}
$$

The numbers $t_{j}, 0 \leq j \leq \min \{m, n\}-1$, are called the superoptimal singular values.

It was shown in [PY1] that for a continuous matrix function $\Phi$ on $\mathbb{T}$ there exists a unique superoptimal approximation $Q$ by analytic functions and

$$
s_{j}(\Phi(\zeta)-Q(\zeta))=t_{j}, \quad \text { a.e. on } \mathbb{T}
$$


(see also $[\operatorname{Tr}]$ where another approach is given). This allows us to consider the operator $\mathcal{A}^{(m, n)}$ of superoptimal approximation by analytic functions which is defined on the space of continuous $m \times n$ functions by $\mathcal{A}^{(m, n)} \Phi \stackrel{\text { def }}{=} Q$. If it does not lead to confusion, we shall write $\mathcal{A}$ for $\mathcal{A}^{(m, n)}$.

In the scalar case $\mathcal{A}=\mathcal{A}^{(1,1)}$ is the operator of best approximation by analytic functions. Its hereditary properties were studied in [CJ], [PK], [To] (see also [Pe3] and $[\mathrm{Pe} 4]$ for continuity and boundedness properties of $\mathcal{A}$ ). It was observed in $[\mathrm{PK}]$ that under natural assumptions a function space $X$ is hereditary for $\mathcal{A}$ (i.e., $\mathcal{A} X \subset X)$ if and only if the recovery problem for unimodular functions is solvable in $X$. Therefore $X$ is hereditary for the operator of best approximation by analytic functions if $X$ is an $\mathcal{R}$-space, $X$ satisfies (A1)-(A4), or $X$ satisfies (B1)-(B3).

In the matrix case heredity properties of the operator $\mathcal{A}^{(m, n)}$ of superoptimal approximation were studied in [PY1]. It was shown in [PY1] that if $X$ satisfies one of the three above conditions and

$$
T_{\bar{\varphi}} f \in X \quad \text { for any } \varphi \in H^{\infty} \text { and any } f \in X_{+},
$$

then $X$ is hereditary for $\mathcal{A}^{(m, n)}$ for any positive integers $m$ and $n$. Throughout the paper for a function space $X, X \subset L^{1}$, we use the following notation:

$$
X_{+} \stackrel{\text { def }}{=}\{f \in X: \hat{f}(j)=0, \text { for } j<0\}, \quad X_{-} \stackrel{\text { def }}{=}\{f \in X: \hat{f}(j)=0, \text { for } j \geq 0\} .
$$

The condition (1.1) does not seem to be satisfactory since it excludes such important spaces like the space $\mathcal{F} \ell^{1}$ of functions with absolutely converging Fourier series.

In this paper we give another approach to the heredity problem which allows us to get rid of the condition (1.1). The approach is based on properties of maximizing vectors of Hankel operators and on the solution of the recovery problem for unitaryvalued functions. Using this approach we show in $\S 9$ that if $X$ is a function space satisfying the axioms (A1)-(A4) or (B1)-(B4), then $X$ is hereditary for the operator $\mathcal{A}^{(m, n)}$ of superoptimal approximation by analytic functions. Note that $\mathcal{R}$-spaces satisfy (1.1) and it was shown in [PY1] that $\mathcal{R}$-spaces are hereditary for $\mathcal{A}^{(m, n)}$.

As we have already mentioned before, our method of solving the recovery problem also brings a new approach to Wiener-Hopf factorizations for $n \times n$ matrix functions in a function space $X$. Recall that if $\Phi$ is a matrix function in $L^{\infty}\left(\mathbb{M}_{n, n}\right)$ such that the Toeplitz operator $T_{\Phi}$ is Fredholm, then by Simonenko's theorem [S], $\Phi$ admits the factorization

$$
\Phi=\Psi_{2}^{*} \Lambda \Psi_{1},
$$

where $\Psi_{1}, \Psi_{1}^{-1}, \Psi_{2}, \Psi_{2}^{-1} \in H^{2}\left(\mathbb{M}_{n, n}\right)$ and $\Lambda$ is a diagonal matrix of the form

$$
\Lambda=\operatorname{diag}\left\{z^{d_{1}}, z^{d_{2}}, \cdots, z^{d_{n}}\right\}, \quad d_{1}, d_{2}, \cdots, d_{n} \in \mathbb{Z}
$$

(see also [LS]). The dimensions of $\operatorname{Ker} T_{\Phi}$ and $\operatorname{Ker} T_{\Phi}^{*}$ are given by

$$
\operatorname{dim} \operatorname{Ker} T_{\Phi}=-\sum_{\left\{j: d_{j}<0\right\}} d_{j}, \quad \operatorname{dim} \operatorname{Ker} T_{\Phi}^{*}=\sum_{\left\{j: d_{j}>0\right\}} d_{j} .
$$

Now let $X$ be a function space such that $X \subset C(\mathbb{T})$, and let $\Phi$ be an $n \times n$ function invertible in $X\left(\mathbb{M}_{n, n}\right)$. We say that $\Phi$ has a Wiener-Hopf factorization in $X$ if $\Phi$ has a representation of the form (1.2), where $\Psi_{1}$ and $\Psi_{2}$ are invertible matrix functions in $X_{+}$. 
Classical results by Plemelj, Muskhelishvili and Vekua solve the Wiener-Hopf factorization problem in Hölder classes $\Lambda_{\alpha}, 0<\alpha<1$ (see [M], [V]). In [GK] the Wiener-Hopf factorization problem was solved in the space $\mathcal{F} \ell^{1}$. In $[\mathrm{G}]$ it was shown that under conditions similar to the axioms (A1)-(A4) each invertible matrix function in $X$ has a Wiener-Hopf factorization in $X$. Budjanu and Gohberg [BG1] considered the general case when the algebra $X$ is not necessarily separable. In [BG2] they prove that the Wiener-Hopf factorization problem is solvable for nonseparable Banach algebras $X$ under certain natural conditions, one of which is that all Hankel operators $H_{\varphi}: X_{+} \rightarrow X_{-}$with scalar symbols $\varphi$ in $X$ are compact (see also $[\mathrm{CG}]$ ). However, those papers did not discuss general conditions under which the above compactness condition holds. In [BG2] this compactness property was established only for the Hölder classes $\Lambda_{\alpha}$ with $0<\alpha<\infty, \alpha \notin \mathbb{Z}$, which gives another proof that each invertible matrix function in $\Lambda_{\alpha}\left(\mathbb{M}_{n, n}\right)$ has a Wiener-Hopf factorization in $\Lambda_{\alpha}$.

In $\S 3$ we prove that if $X$ is a function space satisfying (B1)-(B4), then for any $\varphi \in X$ the Hankel operator $H_{\varphi}: X_{+} \rightarrow X_{-}$is compact. We give in $\S 3$ many examples of nonseparable classical function spaces satisfying (B1)-(B4).

We give a new approach to prove the Wiener-Hopf factorization property in $X$. It is based on studying properties of maximizing vectors of Hankel operators with matrix-valued symbols in $X$. In $\S 4$ we consider the special case of Wiener-Hopf factorizations when the function $\Phi$ is positive definite (so-called Wiener-Masani factorizations). In this case the factor $\Lambda$ in (1.2) is identically equal to $I$. The proof given in $\S 4$ is standard. It is included for the sake of completeness.

Using the results of $\S 4$ we consider in $\S 5$ isometric-outer factorizations of matrix functions in a function space $X$. The results obtained in $\S 5$ allow us to reduce in $\S 8$ the general Wiener-Hopf factorization problem to the case of unitary-valued functions.

The Wiener-Hopf factorization problem for unitary-valued functions in $X$ is solved in $\S 7$ together with the recovery problem. In fact we obtain an even stronger result. We prove that a unitary-valued function $U$ has a Wiener-Hopf factorization in $X$ if we require only that the Toeplitz operator $T_{U}: H^{2}\left(\mathbb{C}^{n}\right) \rightarrow H^{2}\left(\mathbb{C}^{n}\right)$ is Fredholm and $\mathbb{P}_{-} U \in X$.

To study properties of maximizing vectors of Hankel operators with matrixvalued symbols we use general results on symmetric operators on Banach spaces equipped with an inner product (see $\S 2$ ). These results are based on the approach given in $[\mathrm{L}]$.

To conclude the introduction we recall definitions of certain Banach spaces of functions on $\mathbb{T}$.

The Besov class $B_{p}^{s}=B_{p, p}^{s}$ for $1 \leq p \leq \infty$ and $s>0$ can be defined as the space of functions $f$ for which

$$
\left\{2^{|n| s}\left\|f * W_{n}\right\|_{L^{p}}\right\}_{n \in \mathbb{Z}} \in \ell^{p}(\mathbb{Z}),
$$

where the trigonometric polynomials $W_{n}$ can be defined as follows. If $n>0, \hat{W}_{n}$ is a linear function on $\left[2^{n-1}, 2^{n}\right] \cap \mathbb{Z}$ and $\left[2^{n}, 2^{n+1}\right] \cap \mathbb{Z}, \hat{W}_{n}\left(2^{n}\right)=1$, and $\hat{W}_{n}(k)=0$ for $k \notin\left(2^{n-1}, 2^{n+1}\right)$. If $n<0$, then

$$
W_{n} \stackrel{\text { def }}{=} \overline{W_{-n}}, \quad W_{0}(\zeta) \stackrel{\text { def }}{=} \bar{\zeta}+1+\zeta .
$$

The Hölder-Zygmund classes $\Lambda_{\alpha}, 0<\alpha<\infty$, can be defined as $\Lambda_{\alpha}=B_{\infty}^{\alpha}$. 
The space $B M O$ of functions of bounded mean oscillation can be defined as

$$
B M O=\left\{f+\tilde{g}: f, g \in L^{\infty}\right\},
$$

where $\tilde{g}$ is the harmonic conjugate of $g$.

The space $V M O$ of functions of vanishing mean oscillation is the closure of the set of trigonometric polynomials in $B M O$, and can be defined as

$$
V M O=\{f+\tilde{g}: f, g \in C(\mathbb{T})\} .
$$

\section{Symmetric Operators on Banach Spaces With an inNer PROdUCT}

Let $B$ be a complex Banach space with norm $\|\cdot\|_{B}$ which is also equipped with an inner product $(\cdot, \cdot)_{\odot}$ continuous in the norm of $B$. It induces a new norm in $B$ : $\|x\|_{\odot} \stackrel{\text { def }}{=}(x, x)_{\odot}^{1 / 2}, x \in B$.

Suppose that $T$ is a bounded linear operator on $B$ and $T$ is symmetric with respect to the inner product $(\cdot, \cdot)_{\odot}$, i.e.,

$$
(T x, y)_{\odot}=(x, T y)_{\odot}, \quad x, y \in B .
$$

Then $T$ is bounded with respect to the inner product norm $\|\cdot\|_{\odot}$ (see $[\mathrm{L}]$, Theorem I).

Denote by $\mathcal{H}_{\odot}$ the completion of $B$ with respect to the norm $\|\cdot\|_{\odot}$. Clearly, $\mathcal{H}_{\odot}$ is a Hilbert space and we can identify $B$ with a dense subset of $\mathcal{H}_{\odot}$. The operator $T$ extends to $\mathcal{H}_{\odot}$ by continuity. We denote this extension by $T_{\odot}$. Consider the spectra $\sigma(T)$ and $\sigma\left(T_{\odot}\right)$ of the operators $T$ on $B$ and $T_{\odot}$ on $\mathcal{H}_{\odot}$. Then

$$
\sigma\left(T_{\odot}\right) \subset \sigma(T)
$$

(see [L], Theorem II).

Clearly, every eigenvector of $T$ is an eigenvector of $T_{\triangleright}$. We need the following fact.

Lemma 2.1. Let $\lambda$ be an eigenvalue of $T$. Suppose that Range $(T-\lambda I)$ is closed and $\operatorname{dim} \operatorname{Ker}(T-\lambda I)=$ codim Range $(T-\lambda I)$. Then

$$
\operatorname{Ker}(T-\lambda I)=\operatorname{Ker}\left(T_{\odot}-\lambda I\right) .
$$

In [L] Theorem III is stated which asserts that (2.1) holds without any assumption on Range $(T-\lambda I)$. However, the proof given in $[\mathrm{L}]$ does use the assumptions of Lemma 2.1.

Corollary 2.2. Let $\lambda$ be an eigenvalue of $T$. Suppose that there is a compact operator $K$ on $B$ such that the operator $T-\lambda I-K$ is invertible on $B$. Then (2.1) holds.

Proof. Without loss of generality we may assume that $\lambda=0$. Put $R=T-K$. We have

$$
T=R\left(R^{-1} K+I\right)=\left(K R^{-1}+I\right) R .
$$

Clearly, $\operatorname{Ker} T=\operatorname{Ker}\left(R^{-1} K+I\right)$ and Range $T=\operatorname{Range}\left(K R^{-1}+I\right)$. Since $R^{-1} K$ and $K R^{-1}$ are compact it follows that Range $\left(K R^{-1}+I\right)$ is closed and

$$
\begin{aligned}
\operatorname{codim} \operatorname{Range}\left(K R^{-1}+I\right) & =\operatorname{dim} \operatorname{Ker}\left(K R^{-1}+I\right) \\
& =\operatorname{dim} \operatorname{Ker} R\left(K R^{-1}+I\right) \\
& =\operatorname{dim} \operatorname{Ker}\left(R K R^{-1}+R\right) .
\end{aligned}
$$


On the other hand

$$
\operatorname{dim} \operatorname{Ker}\left(R^{-1} K+I\right)=\operatorname{dim} \operatorname{Ker}\left(R^{-1} K+I\right) R=\operatorname{dim} \operatorname{Ker}\left(R K R^{-1}+R\right),
$$

which completes the proof.

Corollary 2.3. Suppose that $T$ is a compact operator on $B$ and $\lambda \neq 0$. Then (2.1) holds.

\section{FUnCtion SPACES AND BEST ANALYTIC APPROXIMATION}

In $[\mathrm{PK}]$ three big classes of function spaces were found that are invariant under the operator $\mathcal{A}$ of best approximation by analytic functions (see also earlier results in [CJ]). Recall that the operator $\mathcal{A}$ is defined on the class $V M O$ of functions of vanishing mean oscillation as follows.

Let $\varphi \in V M O$. Then there exists a unique function $f$ in $B M O A \stackrel{\text { def }}{=} B M O \cap H^{2}$ such that $\varphi-f \in L^{\infty}(\mathbb{T})$ and

$$
\|\varphi-f\|_{\infty}=\inf \left\{\|\varphi-g\|_{\infty}: g \in B M O A, \varphi-g \in L^{\infty}\right\}=\left\|H_{\varphi}\right\| .
$$

We define the operator $\mathcal{A}$ by $\mathcal{A} \varphi \stackrel{\text { def }}{=} f$.

The first class found in $[\mathrm{PK}]$ is the class of $\mathcal{R}$-spaces, i.e., function spaces which can be described in terms of rational approximations in $B M O$. To be more precise, a space $X$ of functions on $\mathbb{T}$ is called an $\mathcal{R}$-space if there is an ideal sequence space $E \subset c_{0}$ (i.e., a linear sequence space with the property

$$
\left.\left\{x_{n}\right\}_{n \geq 0} \in E, \quad\left|y_{n}\right| \leq\left|x_{n}\right|, n \geq 0 \quad \Rightarrow \quad\left\{y_{n}\right\}_{n \geq 0} \in E\right)
$$

such that $\varphi \in X$ if and only if $\varphi \in B M O$ and

$$
\left\{\operatorname{dist}_{B M O}\left(\varphi, \mathcal{R}_{n}\right)\right\}_{n \geq 0} \in E .
$$

Here $\mathcal{R}_{n}$ is the class of rational functions of degree at most $n$ with poles outside $\mathbb{T}$, i.e.,

$$
\mathcal{R}_{n}=\left\{\frac{p}{q} \in L^{\infty}(\mathbb{T}): p, q \text { are polynomials }, \operatorname{deg} p \leq n, \operatorname{deg} q \leq n\right\} .
$$

It follows from the Nehari theorem and from the Adamyan-Arov-Krein theorem (see, e.g., $[\mathrm{N}]$ or $[\mathrm{PK}]$ ) that for a linear $\mathcal{R}$-space $X$ defined by (3.1) a function $\varphi \in B M O$ belongs to $X$ if and only if

$$
\left\{s_{n}\left(H_{\varphi}\right)\right\}_{n \geq 0} \in E, \quad\left\{s_{n}\left(H_{\bar{\varphi}}\right)\right\}_{n \geq 0} \in E
$$

(see $[\mathrm{PK}])$.

It was shown in $[\mathrm{PK}]$ that if $X$ is a linear $\mathcal{R}$-space, then $\mathcal{A} X \subset X$.

If $X$ is a Banach (or a quasi-Banach) $\mathcal{R}$-space (i.e., the corresponding ideal space $E$ is equipped with a norm (or a quasi-norm) satisfying

$$
\left|x_{n}\right| \leq\left|y_{n}\right|, n \geq 0 \quad \Rightarrow \quad\left\|\left\{x_{n}\right\}_{n \geq 0}\right\|_{E} \leq\left\|\left\{y_{n}\right\}_{n \geq 0}\right\|_{E},
$$

and $\|\varphi\|_{X}$ is equivalent to $\left.\left\|\left\{\operatorname{dist}_{B M O}\left(\varphi, \mathcal{R}_{n}\right)\right\}_{n \geq 0}\right\|_{E}\right)$, then the operator $\mathcal{A}$ is bounded on $X$, i.e.,

(see $[\mathrm{PK}])$.

$$
\|\mathcal{A} \varphi\|_{X} \leq \mathrm{const}\|\varphi\|_{X}, \quad \varphi \in X
$$

The spaces $V M O$ and the Besov spaces $B_{p}^{1 / p}, 0<p<\infty$, are $\mathcal{R}$-spaces (see $[\mathrm{PK}],[\mathrm{Pe} 1])$.

The second class of function spaces described in $[\mathrm{PK}]$ is the class of function spaces satisfying the following axioms: 
(A1) If $f \in X$, then $\bar{f} \in X$ and $\mathbb{P}_{+} f \in X$;

(A2) $X$ is a Banach algebra with respect to pointwise multiplication;

(A3) the set of trigonometric polynomials is dense in $X$;

(A4) every complex homomorphism on $X$ is of the form $f \mapsto f(\zeta)$ for some $\zeta \in \mathbb{T}$.

Recall that a complex homomorphism is a nonzero multiplicative linear functional.

It was shown in $[\mathrm{PK}]$ that if $X$ satisfies (A1)-(A4), then $\mathcal{A} X \subset X$. We refer the reader to $[\mathrm{PK}]$ where many examples of spaces satisfying (A1)-(A4) are given. Note that the space $\mathcal{F} \ell^{1}$ of functions with absolutely converging Fourier series and the Besov spaces $B_{p}^{s}$ for $1 \leq p<\infty, s>1 / p$ satisfy (A1)-(A4).

Note, however, that the operator $\mathcal{A}$ is unbounded on $B_{p}^{s}, 1 \leq p<\infty, s>1 / p$ (see $[\mathrm{Pe} 4]$ ), and on $\mathcal{F} \ell^{1}$ (see $[\mathrm{Pa}]$ ).

All function spaces satisfying (A1)-(A4) are separable. The third class of function spaces introduced in $[\mathrm{PK}]$ includes many nonseparable Banach spaces. It consists of function spaces (not necessarily normed spaces) satisfying the following axioms:

(B1) $X$ is an algebra with respect to pointwise multiplication which contains the trigonometric polynomials and such that if $f \in X$, then $\bar{f} \in X$ and $\mathbb{P}_{+} f \in X$;

(B2) there exists a space $Y$ satisfying (A1)-(A4) such that $X \subset Y$ and $T_{\bar{f}} X_{+} \subset$ $X_{+}$for every $f \in Y_{+}$;

(B3) if $f \in X$ and $\inf _{\tau \in \mathbb{T}}|f(\tau)|>0$, then $f^{-1} \in X$.

Note that in $[\mathrm{PK}]$ the axioms (B1)-(B3) were stated in a slightly different way but it can be shown easily that our axioms (B1)-(B3) are equivalent to the axioms (B1)-(B3) stated in [PK]. It was shown in [PK] that if $X$ satisfies (B1)-(B3), then $\mathcal{A} X \subset X$.

In this paper we deal with Banach algebras $X$ which satisfy (B1)-(B3) and the following axiom:

(B4) there exists a Banach space $Z$ such that $X_{+} \subset Z \subset H^{\infty}$, the inclusion $X_{+} \rightarrow Z$ is compact, and $T_{\bar{f}} X_{+} \subset X_{+}$for every $f \in Z$.

Clearly, $X$ satisfies (B1)-(B4) if $X$ is a Banach algebra satisfying (B1), (B3) and the following axiom:

$\left(\mathrm{B} 2^{\prime}\right)$ there exists a space $Y$ satisfying $(\mathrm{A} 1)-(\mathrm{A} 4)$ such that $X \subset Y$, the inclusion $X \rightarrow Y$ is compact, and $T_{\bar{f}} X_{+} \subset X_{+}$for every $f \in Y_{+}$.

We shall see later that many classical Banach spaces satisfy (B1), (B2'), (B3).

It is easy to see that if $X$ satisfies the axioms (A1)-(A4) or $X$ satisfies the axioms (B1)-(B4), then for any $\varphi \in X$ the Hankel operator $H_{\varphi}$ is a bounded operator from $X_{+}$to $X_{-}$. It is also easy to see that if $X$ satisfies (A1)-(A4), then for any $\varphi \in X$, $H_{\varphi}$ is a compact operator from $X_{-}$to $X_{+}$(by (A3) $H_{\varphi}$ is a norm limit of a sequence of Hankel operators with polynomial symbols which have finite rank). Let us prove that the same is true for spaces $X$ satisfying (B1)-(B4).

Theorem 3.1. Let $X$ be a function space satisfying (B1)-(B4) and let $\varphi \in X$. Then the Hankel operator $H_{\varphi}$ is a compact operator from $X_{-}$to $X_{+}$.

Proof. We can assume without loss of generality that $\varphi \in X_{-}$. It follows easily from (B4) that $H_{\varphi} f \in X_{-}$for any $f \in Z$. By the closed graph theorem $H_{\varphi}$ is a bounded operator from $Z$ to $X_{-}$. The result follows now from the fact that the inclusion $X_{+} \rightarrow Z$ is compact. 
It is easy to see and well known that if $X$ satisfies (A1)-(A4), then every complex homomorphism on $X_{+}$coincides with a point evaluation $f \mapsto f(\zeta)$ at some $\zeta \in$ clos $\mathbb{D}$.

Suppose now that $X$ is a Banach algebra satisfying (B1)-(B3). It follows easily from (B2) that $X \subset C(\mathbb{T})$ and so all point evaluations $f \mapsto f(\zeta), \zeta \in \mathbb{T}$, are complex homomorphisms on $X$. The following fact is apparently well known.

Lemma 3.2. Let $X$ be a Banach algebra satisfying (B1)-(B3). Then each complex homomorphism on $X$ has the form $f \mapsto f(\zeta)$ for some $\zeta \in \mathbb{T}$. Each complex homomorphism on $X_{+}$has the form $f \mapsto f(\zeta)$ for some $\zeta \in \operatorname{clos} \mathbb{D}$.

Proof. Let $\omega$ be a complex homomorphism on $X$ which is not a point evaluation at a point of $\mathbb{T}$. Then there are functions $f_{1}, \cdots, f_{n}$ in $X$ such that

$$
\inf _{\zeta \in \mathbb{T}} \sum_{k=1}^{n}\left|f_{k}(\zeta)\right|^{2}>0, \quad \omega\left(f_{k}\right)=0, k=1, \cdots, n .
$$

Put $f=\sum_{k=1}^{n}\left|f_{k}\right|^{2} \in X$. Clearly, $\omega(f)=\sum_{k=1}^{n} \omega\left(\bar{f}_{k}\right) \omega\left(f_{k}\right)=0$. Hence, $f$ is not invertible in $X$, which contradicts (B3).

Suppose now that $\omega$ is a complex homomorphism on $X_{+}$which is not of the form $f \mapsto f(\zeta), \zeta \in \operatorname{clos} \mathbb{D}$. Then there are functions $f_{1}, \cdots, f_{n}$ in $X_{+}$such that

$$
\inf _{\zeta \in \operatorname{clos} \mathbb{D}} \sum_{k=1}^{n}\left|f_{k}(\zeta)\right|^{2}>0, \quad \omega\left(f_{k}\right)=0, k=1, \cdots, n
$$

Since $X_{+} \subset C_{A} \stackrel{\text { def }}{=} C(\mathbb{T}) \cap H^{2}$ and all complex homomorphisms on $C_{A}$ are point evaluations at points of $\operatorname{clos} \mathbb{D}$, there exist $g_{1}, \cdots, g_{n}$ in $C_{A}$ such that $\sum_{k=1}^{n} f_{k} g_{k}=\mathbf{1}$ ( $\mathbf{1}$ is the constant function identically equal to 1 ). Clearly, we can find analytic polynomials $q_{1}, \cdots, q_{n}$ such that

$$
\inf _{\zeta \in \operatorname{clos} \mathbb{D}}\left|\sum_{k=1}^{n} f_{k}(\zeta) q_{k}(\zeta)\right|>0 .
$$

By (B3) the function $h \stackrel{\text { def }}{=}\left(\sum_{k=1}^{n} f_{k} q_{k}\right)^{-1}$ belongs to $X_{+}$. Put $\varphi_{k} \stackrel{\text { def }}{=} h q_{k} \in X_{+}$. Clearly, $\sum_{k=1}^{n} \varphi_{k} f_{k}=\mathbf{1}$, and so $\omega(\mathbf{1})=\sum_{k=1}^{n} \omega\left(\varphi_{k}\right) \omega\left(f_{k}\right)=0$, which is impossible since $\omega(\mathbf{1})=1$.

Let us give examples of nonseparable Banach spaces satisfying the axioms (B1), $\left(\mathrm{B} 2^{\prime}\right),(\mathrm{B} 3)$.

Example 1. The Hölder-Zygmund classes $\Lambda_{\alpha}, \alpha>0$. The space $\Lambda_{\alpha}$ is nonseparable. Denote by $\lambda_{\alpha}$ the closure of the set of trigonometric polynomials in $\Lambda_{a}$. Let $0<\beta<\alpha$. It was shown in [PK] that the classes $\Lambda_{\alpha}$ satisfy the axioms (B1)-(B3) with $Y=\lambda_{\beta}$. Let us show that the inclusion $\Lambda_{\alpha} \rightarrow \lambda_{\beta}$ is compact, and so $\Lambda_{\alpha}$ satisfies (B1), (B2'), and (B3).

Let $W_{n}, n \in \mathbb{Z}$, be the trigonometric polynomials defined in $\S 1$. Define the finite rank operator $R_{m}: \Lambda_{\alpha} \rightarrow \lambda_{\beta}, m \in \mathbb{Z}_{+}$, by

$$
R_{m} f=\sum_{j=-m}^{m} f * W_{j}
$$


It is easy to see that

$$
\begin{aligned}
\left\|f-R_{m} f\right\|_{\lambda_{\beta}} & \leq \sum_{|n| \geq m}\left\|f * W_{n}\right\|_{\lambda_{\beta}} \leq \text { const } \sum_{|n| \geq m} 2^{|n| \beta}\left\|f * W_{n}\right\|_{L^{\infty}} \\
& \leq \text { const } \sum_{|n| \geq m} 2^{|n| \beta} 2^{-|n| \alpha}\left\|f * W_{n}\right\|_{\Lambda_{\alpha}} \\
& \leq \text { const }\|f\|_{\Lambda_{\alpha}} \sum_{|n| \geq m} 2^{|n|(\beta-\alpha)} \rightarrow 0 \quad \text { as } m \rightarrow \infty .
\end{aligned}
$$

Hence, the inclusion $\Lambda_{\alpha} \rightarrow \lambda_{\beta}$ is compact.

Example 2. Let $n$ be a positive integer. Consider the space $B M O^{(n)}$ which consists of functions $f \in L^{\infty}$ whose $n$th derivative (in the distributional sense) is in $B M O$. The space $B M O^{(n)}$ can be endowed with a natural norm. It was shown in $[\mathrm{PK}]$ that $B M O^{(n)}$ satisfies (B1)-(B3) with $Y=\mathcal{F} \ell^{1}$. To show that $B M O^{(n)}$ satisfies $\left(\mathrm{B}^{\prime}\right)$, it is sufficient to prove that the inclusion $B M O^{(n)} \rightarrow \mathcal{F} \ell^{1}$ is compact. Indeed, let $1 / 2<\beta<\alpha<n$. It is well known that $B M O^{(n)} \subset \Lambda_{\alpha}$ and $\lambda_{\beta} \subset \mathcal{F} \ell^{1}$. The result follows now from the fact that the inclusion $\Lambda_{\alpha} \rightarrow \lambda_{\beta}$ is compact (see Example 1).

Example 3. Let $n$ be a positive integer. Consider the space $\left(H^{\infty}+\overline{H^{\infty}}\right)^{(n)}$ of functions $f \in L^{\infty}$ whose $n$th derivative (in the distributional sense) is in $H^{\infty}+\overline{H^{\infty}}$. It was shown in $[\mathrm{PK}]$ that $\left(H^{\infty}+\overline{H^{\infty}}\right)^{(n)}$ satisfies (B1)-(B3) with

$$
Y=\left\{f: \sum_{n \in \mathbb{Z}}|\hat{f}(n)| \log (2+|n|)<\infty\right\}
$$

To prove that $\left(H^{\infty}+\overline{H^{\infty}}\right)^{(n)}$ satisfies the axiom $\left(\mathrm{B} 2^{\prime}\right)$ we show that the inclusion $\left(H^{\infty}+\overline{H^{\infty}}\right)^{(n)} \rightarrow Y$ is compact. Again, let $1 / 2<\beta<\alpha<n$. It is well known that $\left(H^{\infty}+\overline{H^{\infty}}\right)^{(n)} \subset \Lambda_{\alpha}$ and $\lambda_{\beta} \subset Y$. As in Example 2 the result follows from the fact that the inclusion $\Lambda_{\alpha} \rightarrow \lambda_{\beta}$ is compact.

Example 4. Let $w=\left\{w_{n}\right\}_{n \geq 0}$ be a nondecreasing sequence of positive numbers. Consider the space $\mathcal{F} \ell^{\infty}(w)$ to be the space of formal trigonometric series

$$
f \sim \sum_{n \in \mathbb{Z}} \hat{f}(n) z^{n}
$$

such that

$$
\|f\|_{\mathcal{F} \ell^{\infty}(w)}=\sup _{n \in \mathbb{Z}}\left|\hat{f}(n) w_{|n|}\right|<\infty .
$$

Suppose that the sequence $\left\{w_{n}\right\}_{n \geq 0}$ satisfies the following conditions:

$$
\sup _{m \in \mathbb{Z}} \sum_{k+j=m} \frac{w_{|m|}}{w_{|k|} w_{|j|}}<\infty
$$

and

$$
\sup _{n \geq 0} \frac{w_{2 n}}{w_{n}}<\infty .
$$

It was shown in $[\mathrm{PK}]$ that the space $\mathcal{F} \ell^{\infty}(w)$ satisfies (B1)-(B3) with $Y=\mathcal{F} \ell^{1}$. Let us show that the inclusion $\mathcal{F} \ell^{\infty}(w) \rightarrow \mathcal{F} \ell^{1}$ is compact, which would imply that 
$\mathcal{F} \ell^{\infty}(w)$ satisfies $\left(\mathrm{B}^{\prime}\right)$. The following inequality was proved in $[\mathrm{PK}]$ :

$$
\sum_{k=0}^{m} \frac{1}{w_{k}} \leq \sum_{k=0}^{m} \frac{w_{m}}{w_{m-k} w_{k}} \leq \text { const. }
$$

Hence,

$$
\left\|\sum_{|k| \geq n} \hat{f}(k) z^{k}\right\|_{\mathcal{F} \ell^{\infty}(w)} \leq \text { const } \sum_{|k| \geq n} \frac{1}{w_{k}} \rightarrow 0 \quad \text { as } n \rightarrow \infty,
$$

which proves that the inclusion $\mathcal{F} \ell^{\infty}(w) \rightarrow \mathcal{F} \ell^{1}$ is compact.

It is easy to show that the sequence $\left\{w_{n}\right\}_{n \in \mathbb{Z}}$ with $w_{n}=(1+|n|)^{\alpha}, \alpha>1$, satisfies (3.2) and (3.3) and so the space

$$
\left\{f=\sum_{n \in \mathbb{Z}} \hat{f}(n) z^{n}:|\hat{f}(n)| \leq \text { const } \cdot(1+|n|)^{-\alpha}\right\}
$$

satisfies (B1), (B2'), (B3).

Note that it follows from Theorem 3.1 that if $X$ is a space from the above examples and $\varphi \in X$, then the Hankel operator $H_{\varphi}$ is a compact operator from $X_{+}$ to $X_{-}$. For $X=\Lambda_{\alpha}, 0<\alpha<\infty, \alpha \notin \mathbb{Z}$, this fact was proved earlier in [BG2]. However, the proof given in this paper is simpler and works for any $\alpha>0$ as well as for many other function spaces.

Remark. In [Pe3] continuity properties of the operator $\mathcal{A}$ of best approximation by analytic functions as well as of operators of best approximations by meromorphic functions were studied in the norm of a space $X$ satisfying (A1)-(A4). Since by Theorem 3.1 Hankel operators $H_{\varphi}, \varphi \in X$, are compact on $X_{+}$for any $X$ satisfying (B1)-(B4), it follows that the results of [Pe3] are also valid for function spaces satisfying (B1)-(B4).

\section{WienER-MASANi FaCtORIZATIONS}

We consider here the so-called Wiener-Masani factorization problem. Let $W$ be a positive definite square matrix function such that $W \in L^{1}\left(\mathbb{M}_{n, n}\right)$ and $\log \operatorname{det} W \in$ $L^{1}$. It is well known that $W$ admits a factorization in the form

$$
W=\Psi^{*} \Psi,
$$

where $\Psi$ is an outer function in $H^{2}\left(\mathbb{M}_{n, n}\right)$ (see $[R]$ ). Recall that a function $\Psi$ in $H^{2}\left(\mathbb{M}_{n, n}\right)$ is called outer if the set

$$
\left\{\Psi q: q \text { is a polynomial in } H^{2}\left(\mathbb{C}^{n}\right)\right\}
$$

is dense in $H^{2}\left(\mathbb{C}^{n}\right)$. Such factorizations are called Wiener-Masani factorizations. If $W=\Psi_{1}^{*} \Psi_{1}$ is another such factorization with an outer function $\Psi_{1}$, then $\Psi_{1}=U \Psi$, where $U$ is a unitary constant (see $[\mathrm{R}]$ ).

In this section we study Wiener-Masani factorizations for functions $W \in$ $X\left(\mathbb{M}_{n, n}\right)$. Throughout this section $X$ is a function space satisfying the axioms (A1)-(A4) or the axioms (B1)-(B4).

The proof of the following theorem is standard and we give it for completeness.

Theorem 4.1. Let $W$ be a positive definite $n \times n$ matrix function in $X$ such that $W(\zeta)$ is invertible for any $\zeta \in \mathbb{T}$. Then $W$ admits a factorization in the form (4.1), where $\Psi$ is a matrix function invertible in $X_{+}\left(\mathbb{M}_{n, n}\right)$. 
It is easy to see that if $\Psi$ is a function invertible in $X_{+}\left(\mathbb{M}_{n, n}\right)$, then $\Psi$ is outer.

To prove Theorem 4.1 we need a lemma. Note that if $F$ is an $n \times n$ matrix function in $X$, then the Toeplitz operator $T_{F}$ maps $X_{+}\left(\mathbb{C}^{n}\right)$ into itself and can be considered as a bounded operator on $X_{+}\left(\mathbb{C}^{n}\right)$.

Lemma 4.2. Let $W$ be a positive-definite $n \times n$ matrix function in $X$ such that $W(\zeta)$ is invertible for any $\zeta \in \mathbb{T}$. Then the Toeplitz operator $T_{W}$ is an invertible operator on $X_{+}\left(\mathbb{C}^{n}\right)$.

Proof of Lemma 4.2. We have

$$
T_{W} T_{W^{-1}}=I-H_{W}^{*} H_{W^{-1}}
$$

where $H_{W^{-1}}: H^{2}\left(\mathbb{C}^{n}\right) \rightarrow H_{-}^{2}\left(\mathbb{C}^{n}\right)$ is a Hankel operator and $H_{W}^{*}: H_{-}^{2}\left(\mathbb{C}^{n}\right) \rightarrow$ $H^{2}\left(\mathbb{C}^{n}\right)$ is the adjoint to the Hankel operator $H_{W}$. It is easy to see that the operator $H_{W}^{*} H_{W^{-1}}$ maps $X_{+}\left(\mathbb{C}^{n}\right)$ into itself and we can consider it as a bounded operator on $X_{+}\left(\mathbb{C}^{n}\right)$. In fact it is a compact operator on $X_{+}\left(\mathbb{C}^{n}\right)$ since the Hankel operator $H_{W^{-1}}$ is a compact operator from $X_{+}\left(\mathbb{C}^{n}\right)$ to $X_{-}\left(\mathbb{C}^{n}\right)$.

Thus in (4.2) we can assume that both sides of the equation are operators on $X_{+}\left(\mathbb{C}^{n}\right)$. Let us show that $T_{W} T_{W^{-1}}$ is an invertible operator on $X_{+}\left(\mathbb{C}^{n}\right)$. Since $H_{W}^{*} H_{W^{-1}}$ is compact, it is sufficient to show that $\operatorname{Ker} T_{W} T_{W^{-1}}=\{\mathbb{O}\}$. This follows from the fact that $\operatorname{Ker} T_{W}=\operatorname{Ker} T_{W^{-1}}=\{\mathbb{O}\}$.

Let us show that $\operatorname{Ker} T_{W}=\{\mathbb{O}\}$; the proof of the equality $\operatorname{Ker} T_{W^{-1}}=\{\mathbb{O}\}$ is the same. Suppose that $f \in \operatorname{Ker} T_{W}$. We have

$$
\begin{aligned}
0 & =\left(T_{W} f, f\right)_{L^{2}\left(\mathbb{C}^{n}\right)}=\left(\mathbb{P}_{+} W f, f\right)_{L^{2}\left(\mathbb{C}^{n}\right)}=\left(W f, \mathbb{P}_{+} f\right)_{L^{2}\left(\mathbb{C}^{n}\right)} \\
& =(W f, f)_{L^{2}\left(\mathbb{C}^{n}\right)}=\int_{\mathbb{T}}(W(\zeta) f(\zeta), f(\zeta))_{\mathbb{C}^{n}} d \boldsymbol{m}(\zeta) .
\end{aligned}
$$

Since $W(\zeta)$ is positive definite and invertible, it follows that $f=\mathbb{O}$.

It follows now from the invertibility of $T_{W} T_{W^{-1}}$ that $T_{W}$ maps $X_{+}\left(\mathbb{C}^{n}\right)$ onto itself. A similar argument shows that

$$
T_{W^{-1}} T_{W}=I-H_{W^{-1}}^{*} H_{W}
$$

is an invertible operator on $X_{A}\left(\mathbb{C}^{n}\right)$ which implies that the operator $T_{W}$ on $X_{+}\left(\mathbb{C}^{n}\right)$ has trivial kernel.

Proof of Theorem 4.1. Since $T_{W}$ is invertible as an operator on $X_{+}\left(\mathbb{C}^{n}\right)$, there exist functions $f_{j} \in X_{+}\left(\mathbb{C}^{n}\right), 1 \leq j \leq n$, such that

$$
T_{W} f_{j}=\left(\begin{array}{c}
\mathbb{O} \\
\vdots \\
\mathbf{1} \\
\vdots \\
\mathbb{O}
\end{array}\right),
$$

( $\mathbf{1}$ is in the $j$ th row). Consider the $n \times n$ matrix function

$$
F=\left(\begin{array}{lll}
f_{1} & \cdots & f_{n}
\end{array}\right) .
$$

Clearly,

$$
\left(\mathbb{P}_{+} W F\right)(\zeta)=I, \quad \text { a.e. on } \mathbb{T} .
$$

It follows that $\overline{W F} \in H^{2}$. 
Put $\Xi=F^{*} W F$. Then $\Xi \in \overline{H^{1}}$ since both $F^{*}$ and $W F$ are in $\overline{H^{2}}$. Clearly, $\Xi^{*}=\Xi$, and so $\Xi$ is a constant function.

Assume that $\operatorname{det} \Xi=0$. We have $\operatorname{det} \Xi=|\operatorname{det} F(\zeta)|^{2} \operatorname{det} W(\zeta)$ for $\zeta \in \mathbb{T}$. Since the columns $f_{1}(\zeta), \cdots, f_{n}(\zeta)$ of $F(\zeta)$ are linearly independent on $\mathbb{T}$, it follows that $\operatorname{det} F(\zeta) \neq 0$ on $\mathbb{T}$. However, this contradicts the invertibility of $W(z), \zeta \in \mathbb{T}$. Hence det $\Xi \neq 0$, and so $\Xi$ is invertible.

Put $\Psi \stackrel{\text { def }}{=} \Xi^{1 / 2} F^{-1}$. Clearly, $\Psi, \Psi^{-1} \in X$. We have

$$
\Psi^{*} \Psi=F^{*-1} \Xi^{1 / 2} \Xi^{1 / 2} F^{-1}=W .
$$

The last equality is an immediate consequence of the definition of $\Xi$.

Corollary 4.3. Suppose that $W$ satisfies the hypotheses of Theorem 4.1. If $\Psi$ is an outer $n \times n$ matrix function in $H^{2}$ such that $\Psi^{*} \Psi=W$, then $\Psi, \Psi^{-1} \in X$.

Proof. The result follows immediately from Theorem 4.1 and the uniqueness of a Wiener-Masani factorization modulo a constant unitary factor.

Corollary 4.4. Suppose that $W$ satisfies the hypotheses of Theorem 4.1. If $\Omega$ is an outer $n \times n$ matrix function in $H^{2}$ such that $\Omega \Omega^{*}=W$, then $\Omega, \Omega^{-1} \in X$.

Proof. Consider the transposed matrices: $W^{\mathrm{t}}=\left(\Omega^{\mathrm{t}}\right)^{*} \Omega^{\mathrm{t}}$. Then $\Omega^{\mathrm{t}}$ is also an outer function since a square matrix function in $H^{2}$ is outer if and only if its determinant is a scalar outer function (see $[\mathrm{N}]$, Lect. I, Sect. 6). The result follows now from Corollary 4.3.

\section{ISOMETRIC-OUTER FACTORIZATIONS}

In this section we consider representations of matrix functions as a product of an isometric-valued function and an outer function. It is well known that if $\Phi$ is an $n \times m$ matrix function in $L^{2}$ such that $\operatorname{det} \Phi^{*} \Phi \in L^{1}$, then $\Phi$ admits a factorization $\Phi=U \Psi$, where $\Psi$ is an $n \times n$ outer matrix function in $H^{2}$ and $U$ is an $n \times m$ isometric-valued function, i.e., for almost all $\zeta \in \mathbb{T}$

$$
\|U(\zeta) x\|_{\mathbb{C}^{m}}=\|x\|_{\mathbb{C}^{n}}
$$

for any $x \in \mathbb{C}^{n}$. Such a factorization is unique modulo a multiplicative constant.

As usual, we assume here that $X$ is a function space satisfying the axioms (A1)(A4) or the axioms (B1)-(B4). In this section we show that if $\Phi \in X$, then $U \in X$ and $\Psi$ is an invertible function in $X$.

Theorem 5.1. Let $\Phi$ be an $n \times m$ matrix function in $X$ such that $\operatorname{rank} \Phi(\zeta)=n$ for all $\zeta \in \mathbb{T}$. Then $\Phi$ admits a factorization

$$
\Phi=U \Psi,
$$

where $U$ is an $n \times m$ isometric-valued function in $X$ and $\Psi$ is an $n \times n$ outer function such that $\Psi, \Psi^{-1} \in X$.

Proof. Let $W \stackrel{\text { def }}{=} \Phi^{*} \Phi$. Then $W$ is positive definite and $W$ is invertible in $X$. By Theorem 4.1, $W$ admits a factorization

$$
W=\Psi^{*} \Psi,
$$

where $\Psi$ is an invertible function in $X$. Put $U=\Phi \Psi^{-1}$. Clearly, $U \in X$. We have

$$
U^{*} U=\Psi^{*-1} \Phi^{*} \Phi \Psi^{-1}=\Psi^{*-1} \Psi^{*} \Psi \Psi^{-1} \equiv I,
$$

and so $U$ is isometric-valued. 


\section{Maximizing Vectors of Vectorial Hankel operators}

Again, unless otherwise specified, we assume in this section that $X$ is a function space satisfying the axioms (A1)-(A4) or the axioms (B1)-(B4).

The following result shows that for Hankel operators with symbols in $X$ the maximizing vectors also belong to $X$. In the scalar case this was proved earlier in $[\mathrm{PK}]$; see also $[\mathrm{DG}]$ for similar results, though the approach of $[\mathrm{DG}]$ is quite different.

Theorem 6.1. Let $\Phi$ be an $m \times n$ matrix function in $X$ such that $\mathbb{P}_{-} \Phi \neq \mathbb{O}$. Then all maximizing vectors of $H_{\Phi}$ belong to $X$.

Proof. Consider the compact self-adjoint operator $H_{\Phi}^{*} H_{\Phi}$ on $H^{2}\left(\mathbb{C}^{n}\right)$. Obviously, it maps $X_{+}\left(\mathbb{C}^{n}\right)$ into itself. Let $R$ be the operator on $X_{+}\left(\mathbb{C}^{n}\right)$ defined by $R f=$ $H_{\Phi}^{*} H_{\Phi} f, f \in X_{+}\left(\mathbb{C}^{n}\right)$. As we have seen in $\S 3$ (see Theorem 3.1 and a discussion preceding it), the operator $R$ is compact. Since the maximizing vectors of $H_{\Phi}$ are the eigenvectors of $H_{\Phi}^{*} H_{\Phi}$ that correspond to the eigenvalue $\left\|H_{\Phi}\right\|^{2}$, the result follows from Corollary 2.3.

Theorem 6.2. Under the hypotheses of Theorem 6.1 there exists a maximizing vector $f \in X$ such that $f(\zeta) \neq \mathbb{O}$ for any $\zeta \in \mathbb{T}$.

To prove Theorem 6.2 we need the following lemma from [PY3].

Lemma 6.3. Let $X$ be a function space satisfying the axioms (A1)-(A4). Suppose that $\Phi$ is an $m \times n$ function in $X$ such that $\mathbb{P}_{-} \Phi \neq \mathbb{O}$. If $f$ is a maximizing vector of $H_{\Phi}$ and $f(\zeta)=\mathbb{O}$ for some $\zeta \in \mathbb{T}$, then $(\zeta-z)^{-1} f \in X$ and $(\zeta-z)^{-1} f$ is a maximizing vector of $H_{\Phi}$.

Note that in [PY3] it was assumed that $X$ satisfies (A1)-(A4) and condition (1.1). However, (1.1) was used in [PY3] only to conclude that the maximizing vectors belong to $X$. Since this is true by Theorem 6.1 without assuming (1.1), the proof of Lemma 6.3 given in [PY3] works for spaces $X$ satisfying (B1)-(B4).

Proof of Theorem 6.2. Suppose first that $X$ satisfies (A1)-(A4). Take an arbitrary maximizing vector $f_{0}$. If $f_{0}\left(\zeta_{0}\right)=\mathbb{O}$ for some $\zeta_{0} \in \mathbb{T}$, consider the function $f_{1} \stackrel{\text { def }}{=}$ $\left(\zeta_{0}-z\right)^{-1} f_{0}$. By Lemma $6.3, f_{1}$ is also a maximizing vector for $H_{\Phi}$. If $f_{1}\left(\zeta_{1}\right)=\mathbb{O}$ for some $\zeta_{1} \in \mathbb{T}$, consider the maximizing vector $f_{2} \stackrel{\text { def }}{=}\left(\zeta_{1}-z\right)^{-1} f_{1}$. Since the operator $H_{\Phi}$ is compact, there can be only finitely many linear independent maximizing vectors. Therefore our process cannot last infinitely long, which means that sooner or later we construct a maximizing vector $f$ such that $f(\zeta) \neq \mathbb{O}$ for any $\zeta \in \mathbb{T}$.

Suppose now that $X$ satisfies the axioms (B1)-(B4). Let $Y$ be the space from (B2). Clearly, $\Phi \in Y$, and so there exists a maximizing vector $f \in Y$ such that $f(\zeta) \neq 0$ for any $\zeta \in \mathbb{T}$. It follows from Theorem 6.1 that $f \in X$.

\section{Unitary-Valued functions. The ReCOVERY PRoblem}

In this section we use the results of $\S 6$ to solve two problems: the Wiener-Hopf factorization problem for unitary-valued functions and the recovery problem for unitary-valued functions.

We stated the recovery problem in the introduction under the assumption that the Toeplitz operator $T_{U}: H^{2}\left(\mathbb{C}^{n}\right) \rightarrow H^{2}\left(\mathbb{C}^{n}\right)$ is Fredholm or has a dense range. 
If $T_{U}$ is Fredholm, then $T_{\bar{z}^{N} U}$ maps $\left.H^{2}\left(\mathbb{C}^{n}\right)\right)$ onto itself for sufficiently large $N$. Therefore it is sufficient to consider the case when $T_{U}$ has dense range.

Let us start with the recovery problem for a Banach (or quasi-Banach) $\mathcal{R}$-space $X$ (see $\S 3$ ). It was shown in [Pe2] that if $U$ is a unitary-valued function such that $T_{U}$ has dense range in $H^{2}\left(\mathbb{C}^{n}\right)$, then $H_{U^{*}}^{*} H_{U^{*}}$ is unitarily equivalent to the restriction of $H_{U}^{*} H_{U}$ to an invariant subspace. It follows that $s_{k}\left(H_{U^{*}}\right) \leq s_{k}\left(H_{U}\right)$ for any $k \geq 0$. This implies the following solution of the hereditary problem for $\mathcal{R}$-spaces.

Theorem 7.1. Let $X$ be a quasi-Banach $\mathcal{R}$-space and let $U$ be a unitary-valued matrix function such that the Toeplitz operator $T_{U}: H^{2}\left(\mathbb{C}^{n}\right) \rightarrow H^{2}\left(\mathbb{C}^{n}\right)$ has dense range. If $\mathbb{P}_{-} U \in X$, then $U \in X$.

Let us proceed to the case when $X$ satisfies the axioms (A1)-(A4) or the axioms (B1)-(B4).

Theorem 7.2. Suppose that $X$ is a function space that satisfies the axioms (A1)(A4) or the axioms (B1)-(B4). Let $U$ be a unitary-valued matrix function such that the Toeplitz operator $T_{U}$ has dense range in $H^{2}\left(\mathbb{C}^{n}\right)$. If $\mathbb{P}_{-} U \in X$, then $U \in X$.

Let us first show that under the hypotheses of Theorem 7.2 the operator $T_{U}$ : $H^{2}\left(\mathbb{C}^{n}\right) \rightarrow H^{2}\left(\mathbb{C}^{n}\right)$ is Fredholm. Since $X \subset C(\mathbb{T})$, it follows that $\mathbb{P}_{-} U \in V M O$. As we have mentioned in $\S 3, V M O$ is an $\mathcal{R}$-space, and so by Theorem 7.1, $U \in V M O$. Therefore the Hankel operators $H_{U}$ and $H_{U^{*}}$ are compact (see, e.g., [PK]) and the fact that $T_{U}$ is Fredholm follows from the following well-known formulas which are easy to verify:

$$
I-T_{U^{*}} T_{U}=H_{U}^{*} H_{U}, \quad I-T_{U} T_{U^{*}}=H_{U^{*}}^{*} H_{U^{*}} .
$$

Now let $U$ be a unitary-valued function such that $T_{U}$ is Fredholm. Then by Simonenko's theorem mentioned in the introduction, $U$ admits a factorization

$$
U=\Psi_{2}^{*} \Lambda \Psi_{1}
$$

where $\Psi_{1}^{ \pm 1}, \Psi_{2}^{ \pm 1} \in H^{2}\left(\mathbb{C}^{n}\right)$ and $\Lambda$ is a diagonal matrix of the form

$$
\Lambda=\operatorname{diag}\left\{z^{d_{1}}, z^{d_{2}}, \cdots, z^{d_{n}}\right\}, \quad d_{1}, \cdots, d_{n} \in \mathbb{Z} .
$$

It is easy to see that Theorem 7.2 follows immediately from the following theorem which solves the Wiener-Hopf factorization problem in $X$ for unitary-valued functions. In fact we obtain an even stronger result since instead of assuming that $U \in X$ we assume only that $\mathbb{P}_{-} U \in X$.

Theorem 7.3. Suppose that $X$ is a function space that satisfies the axioms (A1)(A4) or the axioms (B1)-(B4). Let $U$ be a unitary-valued function such that $\mathbb{P}_{-} U \in X$. Then the functions $\Psi_{1}, \Psi_{1}^{-1}, \Psi_{2}$, and $\Psi_{2}^{-1}$ in (7.1) belong to $X$.

Proof. Let us first prove that $\Psi_{1} \in X$ and $\Psi_{1}^{-1} \in X$. Without loss of generality we may assume that all the indices of the factorization (7.1) are negative. Otherwise, we can multiply $U$ by $\bar{z}^{N}$ for a sufficiently large integer $N$. Let $e$ be an arbitrary nonzero vector in $\mathbb{C}^{n}$. It is easy to see that $\Psi_{1}^{-1} e$ is a maximizing vector for $H_{U}$. It follows now from Theorem 6.1 that $\Psi_{1}^{-1} e \in X$, and so $\Psi_{1}^{-1} \in X$.

Let us show that $\Psi_{1} \in X$. Put $G=\Psi_{1}^{-1} \in X$. It is sufficient to show that the matrix $G(\zeta)$ is invertible for all $\zeta \in \mathbb{T}$. 
Consider $\operatorname{Ker} T_{U}$. It admits the following description:

$$
\operatorname{Ker} T_{U}=\left\{G\left(\begin{array}{c}
q_{1} \\
\vdots \\
q_{n}
\end{array}\right): q_{j} \text { is a polynomial in } H^{2}, \operatorname{deg} q_{j}<\left|d_{j}\right|\right\} .
$$

Indeed, it is easy to see that the right-hand side of (7.2) is contained in the left-hand side and by (1.3) the dimensions of both sides are equal.

Assume that $G\left(\zeta_{0}\right)$ is noninvertible for some $\zeta_{0} \in \mathbb{T}$. Then $G\left(\zeta_{0}\right) e=\mathbb{O}$ for some nonzero $e \in \mathbb{C}^{n}$. It is easy to see from (7.2) that $G e$ is a nonzero function in Ker $T_{U}$. Clearly, a nonzero vector in $H^{2}\left(\mathbb{C}^{n}\right)$ is maximizing for $H_{U}$ if and only if it belongs to $\operatorname{Ker} T_{U}$.

If $X$ satisfies (A1)-(A4), then by Lemma 6.3 the function $\left(\zeta_{0}-z\right)^{-1} G e$ is a maximizing vector for $H_{U}$, and so it belongs to $\operatorname{Ker} T_{U}$. If $X$ satisfies (B1)-(B4), then $G e \in Y$, where $Y$ is the space from the axiom (B2). Again, by Lemma 6.3 $\left(\zeta_{0}-z\right)^{-1} G e \in Y$ and $\left(\zeta_{0}-z\right)^{-1} G e$ is a maximizing vector for $H_{U}$. However, it is easy to see that $\left(\zeta_{0}-z\right)^{-1} G e$ does not belong to the right-hand side of (7.2).

Let us show that $\Psi_{2}, \Psi_{2}^{-1} \in X$. Since $U$ is unitary-valued, it follows that

$$
\left(U^{*} U\right)(\zeta)=\left(\Psi_{1}^{*} \Lambda^{*} \Psi_{2} \Psi_{2}^{*} \Lambda \Psi_{1}\right)(\zeta)=I, \quad \zeta \in \mathbb{T},
$$

and so

$$
\Psi_{2} \Psi_{2}^{*}=\Lambda \Psi_{1}^{*-1} \Psi_{1}^{-1} \Lambda^{*} .
$$

Since $\Psi_{2}$ is an outer function and the right-hand side of (7.3) is invertible in $X$, the result follows from Corollary 4.4.

\section{WiEnER-HOPF FACTORIZATIONS}

In this section we consider Wiener-Hopf factorizations of matrix functions in a function space $X$ which satisfies the axioms (A1)-(A4) or the axioms (B1)-(B4). If $\Phi$ is an $n \times n$ matrix function in $X$ such that $\operatorname{det} \Phi(\zeta) \neq 0$ for any $\zeta$ in $\mathbb{T}$, then the Toeplitz operator $T_{\Phi}$ on $H^{2}\left(\mathbb{C}^{n}\right)$ is Fredholm, and so $\Phi$ admits a Wiener-Hopf factorization

$$
\Phi=\Psi_{2}^{*} \Lambda \Psi_{1}
$$

where $\Psi_{1}, \Psi_{1}^{-1}, \Psi_{2}, \Psi_{2}^{-1} \in H^{2}$ and $\Lambda$ is a diagonal matrix function of the form

$$
\Lambda=\operatorname{diag}\left\{z^{d_{1}}, z^{d_{2}}, \cdots, z^{d_{n}}\right\}, \quad d_{1}, \cdots, d_{n} \in \mathbb{Z} .
$$

We are going to prove in this section that for $\Phi \in X$ the matrix functions $\Psi_{1}$ and $\Psi_{2}$ are invertible functions in $X$.

Theorem 8.1. Let $\Phi$ be an $n \times n$ matrix function in $X$ such that $\operatorname{det} \Phi(\zeta) \neq 0$ for any $\zeta$ in $\mathbb{T}$. Suppose that (8.1) is a Wiener-Hopf factorization of $\Phi$. Then $\Psi_{1}, \Psi_{1}^{-1}, \Psi_{2}, \Psi_{2}^{-1} \in X$.

Proof. By Theorem 5.1, $\Phi$ admits a representation $\Phi=U \Psi$, where $U$ is a unitaryvalued function in $X$ and $\Psi$ is an outer function which is invertible in $X$.

Suppose that $\Phi$ admits a factorization in the form (8.1). We have

$$
U=\Psi_{2}^{*} \Lambda \Psi_{1} \Psi^{-1} .
$$

Clearly, $\Psi_{1} \Psi^{-1} \in H^{2}$ and $\left(\Psi_{1} \Psi^{-1}\right)^{-1} \in H^{2}$. By Theorem 7.2, $\Psi_{2}, \Psi_{2}^{-1} \in X$ and $\Psi_{1} \Psi^{-1},\left(\Psi_{1} \Psi^{-1}\right)^{-1} \in X$, which implies that $\Psi_{1}, \Psi_{1}^{-1} \in X$. 


\section{SUPEROPTIMAL APPROXIMATION BY ANALYTIC MATRIX FUNCTIONS}

In this section we study hereditary properties of the operator $\mathcal{A}=\mathcal{A}^{(m, n)}$ of superoptimal approximation by analytic matrix functions. For a function space $X$ satisfying (A1)-(A4) or (B1)-(B4) we are going to prove that if $\Phi$ is an $m \times n$ matrix function in $X$, then $\mathcal{A} \Phi \in X$.

Let us recall how to construct the unique superoptimal approximation to $\Phi \in$ $H^{\infty}+C$ (see [PY2]). Without loss of generality we may assume that $n \leq m$. Let $v$ be a maximizing vector of the Hankel operator $H_{\Phi}: H^{2}\left(\mathbb{C}^{n}\right) \rightarrow H_{-}^{2}\left(\mathbb{C}^{m}\right)$. If $n=1$, then $v$ is a scalar function and $\Phi$ has a unique best approximant $Q$ by $H^{\infty}$ functions which is given by

$$
Q=\Phi-\frac{H_{\Phi} v}{v}
$$

Suppose now that $n>1$. If $\mathbb{P}_{-} \Phi=\mathbb{O}$, then $\mathcal{A} \Phi=\Phi$. Otherwise put $w=\frac{1}{t_{0}} H_{\Phi} v$, where $t_{0} \stackrel{\text { def }}{=}\left\|H_{\Phi}\right\|$. Then the vector functions $v$ and $\bar{z} \bar{w}$ admit the factorizations

$$
v=b_{1} h v_{(\mathrm{i})}, \quad \bar{z} \bar{w}=b_{2} h w_{(\mathrm{i})},
$$

where $h$ is a scalar outer function in $H^{2}, b_{1}$ and $b_{2}$ are finite Blaschke products, and $v_{(\mathrm{i})}$ and $w_{(\mathrm{i})}$ are column functions which are inner and co-outer.

Recall that a matrix function $F \in H^{\infty}\left(\mathbb{M}_{m, n}\right)$ is called inner if the matrices $F(\zeta)$ are isometric for almost all $\zeta \in \mathbb{T}$. $F$ is called outer if multiplication by $F$ on $H^{2}\left(\mathbb{C}^{n}\right)$ has dense range in $H^{2}\left(\mathbb{C}^{m}\right)$. This definition is consistent with the definition given in $\S 4$. Finally, $F$ is called co-outer if the transposed function $F^{\mathrm{t}}$ is outer.

The column functions $v_{(\mathrm{i})}$ and $w_{(\mathrm{i})}$ admit thematic completions, i.e., there exist inner co-outer matrix functions $\Theta$ and $\Xi$ such that the matrix functions $\left(v_{(\mathrm{i})} \bar{\Theta}\right)$ and $\left(\begin{array}{ll}w_{(\mathrm{i})} & \bar{\Xi}\end{array}\right)$ on $\mathbb{T}$ are unitary-valued. We call such functions thematic matrix functions. Put

$$
V=\left(\begin{array}{ll}
v_{(\mathrm{i})} & \bar{\Theta}
\end{array}\right), \quad W=\left(\begin{array}{ll}
w_{(\mathrm{i})} & \bar{\Xi}
\end{array}\right)^{\mathrm{t}} .
$$

If $Q \in H^{\infty}$ is an arbitrary $H^{\infty}$ best approximation to $\Phi$, then $\Phi-Q$ admits the factorization

$$
\Phi-Q=W^{*}\left(\begin{array}{cc}
t_{0} u_{0} & \mathbb{0} \\
\mathbb{0} & \Phi^{(1)}
\end{array}\right) V^{*},
$$

where $u_{0}=\bar{z} \bar{b}_{1} \bar{b}_{2} \bar{h} / h$ and $\Phi^{(1)}$ is an $(m-1) \times(n-1)$ matrix function in $H^{\infty}+C$.

This procedure reduces finding $\mathcal{A} \Phi$ to finding $\mathcal{A} \Phi^{(1)}$. Indeed,

$$
\Phi-\mathcal{A} \Phi=W^{*}\left(\begin{array}{cc}
t_{0} u_{0} & \mathbb{O} \\
\mathbb{O} & \Phi^{(1)}-\mathcal{A} \Phi^{(1)}
\end{array}\right) V^{*}
$$

(see $[\mathrm{PY} 1])$. Continuing in this way we eventually arrive at the situation when $n=1$.

Let $Q$ be an arbitrary $H^{\infty}$ best approximant to $\Phi$. Then it is well known (see, e.g., $[\mathrm{AAK}])$ that

$$
H_{\Phi} v=(\Phi-Q) v=t_{0} w, \quad H_{\Phi}^{*} w=\left(\Phi^{*}-Q^{*}\right) w=t_{0} v,
$$

and so

$$
Q v=\Phi v-t_{0} w=\mathbb{P}_{+} \Phi v, \quad Q^{*} w=\Phi^{*} w-t_{0} v=\mathbb{P}_{-} \Phi^{*} w
$$

Thus $Q$ must satisfy the following equations:

$$
Q v=T_{\Phi} v, \quad Q^{\mathrm{t}}(\bar{z} \bar{w})=T_{\Phi^{\mathrm{t}}}(\bar{z} \bar{w}) .
$$


It follows that

$$
Q v_{(\mathrm{i})}=\varphi_{1}, \quad Q^{\mathrm{t}} w_{(\mathrm{i})}=\varphi_{2},
$$

where

$$
\varphi_{1} \stackrel{\text { def }}{=} \frac{\bar{b}_{1} T_{\Phi} v}{h}, \quad \varphi_{2} \stackrel{\text { def }}{=} \frac{\bar{b}_{2} T_{\Phi^{\mathrm{t}}}(\bar{z} \bar{w})}{h} .
$$

Clearly, (9.6) implies that $\varphi_{1}, \varphi_{2} \in H^{\infty}$. It also follows from (9.6) that

$$
\varphi_{2}^{\mathrm{t}} v_{(\mathrm{i})}=w_{(\mathrm{i})}^{\mathrm{t}} \varphi_{1}
$$

since both functions are equal to $w_{(\mathrm{i})}^{\mathrm{t}} Q v_{(\mathrm{i})}$.

The situation seems to be not quite satisfactory since to lower the size of the matrix function we have to find a best approximant $Q$. To prove that $\mathcal{A} \Phi$ inherits properties of $\Phi$ we should find a best approximant $Q$ in the same class $X$, which looks like a complicated problem.

Fortunately, we do not have to find a best approximant $Q$ in $X$. As observed in [PY2] (see also [PY3]) the above scheme works if $Q$ is not necessarily a best $H^{\infty}$ approximation to $\Phi$. It is sufficient to take an arbitrary function $Q \in H^{\infty}\left(\mathbb{M}_{m . n}\right)$ which satisfies (9.6).

In [PY2] a solution $Q$ of equations (9.6) was found in terms of solutions of corona problems. Since the column functions $v_{(\mathrm{i})}$ and $w_{(\mathrm{i})}$ are inner co-outer and belong to $V M O$ (see [PY1]), it follows that they satisfy Carleson's corona condition (see [PY1]), and so there exist column functions $\psi_{1}$ and $\psi_{2}$ in $H^{\infty}$ such that

$$
\psi_{1}^{\mathrm{t}} v_{(\mathrm{i})}=\psi_{2}^{\mathrm{t}} w_{(\mathrm{i})}=\mathbf{1},
$$

where $\mathbf{1}$ is the function identically equal to 1 . It was found in [PY2] (this is an elementary exercise) that the $H^{\infty}\left(\mathbb{M}_{m, n}\right)$ function

$$
Q=\varphi_{1} \psi_{1}^{\mathrm{t}}+\psi_{2} \varphi_{2}^{\mathrm{t}}-\psi_{2} \varphi_{2}^{\mathrm{t}} v_{(\mathrm{i})} \psi_{1}^{\mathrm{t}}
$$

satisfies equations (9.7), and so a factorization of the form (9.4) holds which allows us to reduce the problem to finding the superoptimal approximant to $\Phi^{(1)}$.

To obtain a heredity theorem for matrix functions $\Phi$ in $X$ we need the following result proved in $[\mathrm{Pe} 5]$.

Lemma 9.1. Let $U$ be an $n \times n$ unitary-valued matrix function of the form $(\vartheta \bar{\Theta})$, where $\vartheta$ is a column function, and the functions $\vartheta$ and $\Theta$ are inner and co-outer. Then the Toeplitz operator $T_{U}: H^{2}\left(\mathbb{C}^{n}\right) \rightarrow H^{2}\left(\mathbb{C}^{n}\right)$ has trivial kernel and dense range.

Theorem 9.2. Suppose that $X$ is a function space satisfying (A1)-(A4) or (B1)(B4). Let $\Phi$ be a matrix function in $X$. Then $\mathcal{A} \Phi \in X$.

Proof. By Theorem 6.2 we can find a maximizing vector $v$ of $H_{\Phi}$ which belongs to $X$ and such that $v(\zeta) \neq \mathbb{O}$ for any $\zeta \in \mathbb{T}$.

We argue by induction on $n$. If $n=1$, the result follows from (9.1). Suppose now that $n>1$.

Clearly, the vector function $w=H_{\Phi} v$ belongs to $X$. Since the outer function $h$ in (9.2) satisfies

$$
|h(\zeta)|=\|v(\zeta)\|_{\mathbb{C}^{n}}=\|w(\zeta)\|_{\mathbb{C}^{m}}
$$

and $v$ has no zeros on $\mathbb{T}$, it follows that $h$ and $h^{-1}$ belong to $X$. Therefore the inner column functions $v_{(\mathrm{i})}$ and $w_{(\mathrm{i})}$ also belong to $X$. Consider the functions $V$ 
and $W$ defined by (9.3). Clearly, $\mathbb{P}_{-} V, \mathbb{P}_{-} W \in X$. It follows from Lemma 9.1 and from Theorem 7.2 that $V, W \in X$. It is also clear that $u_{0} \in X$.

If we show that there exists a solution $Q \in X_{+}\left(\mathbb{M}_{m, n}\right)$ of equations (9.6), it will follow that $\Phi^{(1)} \in X$ and we reduce the problem to the $(m-1) \times(n-1)$ matrix function $\Phi^{(1)} \in X$.

Since $v_{(\mathrm{i})}$ and $w_{(\mathrm{i})}$ belong to $X_{+}$and have no zeros in clos $\mathbb{D}$, it follows from Lemma 3.2 that there exist column functions $\psi_{1}$ and $\psi_{2}$ in $X$ satisfying (9.9). Since the functions $\varphi_{1}$ and $\varphi_{2}$ defined by (9.7) belong to $H^{\infty}$, it follows that they belong to $X_{+}$. It is easy to see now that the function $Q$ defined by (9.10) satisfies (9.6) and belongs to $X_{+}$.

Now we are going to improve the results of [PY3] on continuity properties of the operator $\mathcal{A}$ of superoptimal approximation. Let us return to the procedure of constructing $\mathcal{A} \Phi$ which has been described in the beginning of this section. We have reduced the problem of constructing $\mathcal{A} \Phi$ to the problem of constructing $\mathcal{A} \Phi^{(1)}$. We can proceed with $\Phi^{(1)}$ in the same way as with $\Phi$. Eventually this leads to the following factorization for $\Phi-\mathcal{A} \Phi$ :

$$
\Phi-\mathcal{A} \Phi=W_{0}^{*} W_{1}^{*} \cdots W_{n-1}^{*} D V_{n-1}^{*} V_{n-2}^{*} \cdots V_{0}^{*},
$$

where $D$ is an $m \times n$ matrix function of the form

$$
D=\left(\begin{array}{cccc}
t_{0} u_{0} & \mathbb{O} & \cdots & \mathbb{O} \\
\mathbb{O} & t_{1} u_{1} & \cdots & \mathbb{O} \\
\vdots & \vdots & \ddots & \vdots \\
\mathbb{O} & \mathbb{O} & \cdots & t_{n-1} u_{n-1} \\
\mathbb{O} & \mathbb{O} & \cdots & \mathbb{O} \\
\vdots & \vdots & \ddots & \vdots \\
\mathbb{O} & \mathbb{O} & \cdots & \mathbb{O}
\end{array}\right)
$$

with some unimodular functions $u_{0}, \cdots, u_{n-1} \in X$,

$$
W_{j}=\left(\begin{array}{cc}
I_{j} & \stackrel{\mathbb{O}}{ } \\
\mathbb{O} & \breve{W}_{j}
\end{array}\right), \quad V_{j}=\left(\begin{array}{cc}
I_{j} & \mathbb{0} \\
\mathbb{O} & \breve{V}_{j}
\end{array}\right), \quad 1 \leq j \leq n-1,
$$

and $W_{0}^{\mathrm{t}}, \breve{W}_{j}^{\mathrm{t}}, V_{0}$ and $\breve{V}_{j}$ are thematic functions for $1 \leq j \leq n-1$. Here $I_{j}$ stands for the constant function identically equal to the $j \times j$ identity matrix. We call (9.11) a thematic factorization of $\Phi-\mathcal{A} \Phi$. The numbers $t_{0}, \cdots, t_{n-1}$ are the superoptimal singular values of $\Phi$. If $t_{j} \neq 0$, we define the index $k_{j}$ of the thematic factorization (9.11) by $k_{j}=\mid$ wind $u_{j} \mid$. Note that the winding number wind $u_{j}$ of $u_{j}$ with respect to the origin is always negative.

Theorem 9.2 allows us to improve earlier results of [PY3].

Theorem 9.3. Suppose that $X$ is a function space satisfying (A1)-(A4) or (B1)(B4). Let $\Phi$ be an $m \times n$ matrix function in $X, n \leq m$. Suppose that $t_{n-1} \neq 0$ and all indices $k_{0}, \cdots, k_{n-1}$ of a thematic factorization (9.11) are equal to 1 . Then $\Phi$ is a continuity point of the operator $\mathcal{A}$ in $X\left(\mathbb{M}_{m, n}\right)$.

Theorem 9.4. Suppose that $X$ is a function space satisfying (A1)-(A4) or (B1)(B4). Let $\Phi$ be an $n \times n$ matrix function in $X$ such that the superoptimal singular value $t_{n-1}$ of $\Phi$ is nonzero. If $\mathcal{A}$ is continuous at $\Phi$ in $X\left(\mathbb{M}_{n, n}\right)$, then all indices in any thematic factorization of $\Phi-\mathcal{A} \Phi$ are equal to 1 . 
Using Theorem 9.2, one can prove Theorems 9.3 and 9.4 in exactly the same way Theorems 1.1 and 2.2 were proved in [PY3].

\section{REFERENCES}

[AAK] V.M. Adamyan, D.Z. Arov and M.G. Krein, Infinite Hankel block matrices and some related continuation problems, Izv. Akad. Nauk Armyan. SSR Ser. Mat 6 (1971), 87-112. MR 45:7506

[BG1] M.S. Budjanu And I.C. GohBerg, General theorems on the factorization of matrix valued functions, I. The fundamental theorem, Mat. Issled. 3 (1968), no. 2(8), 87-103 (Russian); English transl., Amer. Math. Soc. Transl. (2) 102 (1973), 1-14. MR 41:4246a

[BG2] M.S. BudJAnu AND I.C. GohBERG, General theorems on the factorization of matrix valued functions, II. Some tests and their consequences, Mat. Issled. 3 (1968), no. 3(8), 3-18 (Russian); English transl., Amer. Math. Soc. Transl. (2) 102 (1973), 15-26. MR 41:4246b

[CG] K. Clancey and I. Gohberg, Factorization of matrix functions and singular integral operators, Oper. Theory: Advances and Appl., 3, Birkhäuser Verlag, Basel-Boston-Stuttgart, 1981. MR 84a:47016

[CJ] L. Carleson and S. Jacobs, Best uniform approximation by analytic functions, Ark. Mat. 10 (1972), 219-229. MR 48:772

[DG] H. Dym And I. C. Gohberg, Unitary interpolants, factorization indices and infinite Hankel block matrices, J. Funct. Anal. 54 (1983), 229-289. MR 85m:47021a

[G] I.C. Gohberg, The factorization problem in normed rings, functions of isometric and symmetric operators and singular integral equations, Uspekhi Mat. Nauk 19:1 (1964), 71124 (Russian); English transl., Russian Math. Surveys 19:1 (1964), 63-114. MR 29:487

[GK] I.C. Gohberg And M.G. Krein, Systems of integral equations on a half line with kernels depending on the difference of arguments, Uspekhi Mat. Nauk 13:2 (1958), 3-72 (Russian); English transl., Amer. Math. Soc. Transl. 14 (1960), 217-287. MR 21:1506

[L] P.B. Lax, Symmetrizable linear transformations, Comm. Pure Appl. Math. 7 (1954), 633-647. MR 16:832d

[LS] G.S. Litvinchuk And I.M. Spitkovski, Factorization of measurable matrix functions, Oper. Theory: Advances and Appl., 25, Birkhäuser Verlag, Basel-Boston, MA, 1987. MR 90g: 47030

[M] N.I. Muskhelishvili, Singular integral equation. Boundary problems of function theory and their application to mathematical physics, 2nd Ed., Fizmatgiz, Moscow, 1962 (Russian); English transl. of 1st ed., Nordhoff, Groningen, 1953. MR 15:434e

[N] N.K. NikOL'SkiI, Treatise on the shift operator. Spectral function theory, Springer-Verlag, Berlin-Heidelberg-New York-Tokyo, 1986. MR 87i:47042

[Pa] M. Papadimitrakis, On best uniform approximation by bounded analytic functions, Bull. London Math. Soc. 28 (1996), 15-18. MR 96j:30054

[Pe1] V.V. Peller, A description of Hankel operators of class $\mathfrak{S}_{p}$ for $p>0$, investigation of the rate of rational approximation and other applications, Mat. Sb. 122 (1983), 481-510; English transl., Math. USSR-Sb. 50 (1985), 465-494. MR 85g:47041

[Pe2] V.V. Peller, Hankel operators and multivariate stationary processes, Proc. Symp. Pure Math. 51 (1990), 357-371. MR 91m:47034

[Pe3] V.V. Peller, Hankel operators and continuity properties of best approximation operators, Algebra i Analiz 2:1 (1990), 163-189; English transl. in Leningrad Math. J. 2 (1991), 139160. MR 91e: 41043

[Pe4] V.V. Peller, Boundedness properties of the operators of best approximation by analytic and meromorphic functions, Ark. Mat. 30 (1992), 331-343. MR 95f:46038

[Pe5] V.V. Peller, Approximation by analytic operator-valued functions, Harmonic analysis and operator theory (Caracas, 1994), 431-448, Contemp. Math., 189, Amer. Math. Soc., Providence, RI, 1995. MR 96i:47048

[PK] V.V. Peller and S.V. Khruschev, Hankel operators, best approximation and stationary Gaussian processes, Russian Math. Surveys 37 (1982), 53-124. MR 84e:47036

[PY1] V.V. Peller And N.J. Young, Superoptimal analytic approximations of matrix functions, J. Functional Analysis 120 (1994), 300-343. MR 94m:47030

[PY2] V.V. Peller And N.J. Young, Construction of superoptimal approximation, Math. Control Signals Systems 8 (1995), 118-137. 
[PY3] V.V. Peller and N.J. Young, Continuity properties of best analytic approximation, J. Reine Angew. Math. 483 (1997), 1-22. MR 97m:47031

[R] Yu.A. Rozanov, Stationary stochastic processes, Fizmatgiz, Moscow, 1963 (Russian). MR 28:2580

[S] I.B. Simonenko, Some general problems of the theory of the Riemann boundary-value problem, Izv. Akad. Nauk SSSR, Ser. Mat. 32 (1968), 1138-1146 (Russian). MR 38:3447

[To] V.A. Tolokonnikov, Generalized Douglas algebras, Algebra i Analiz 3 (1991), 231-252 (Russian). MR 92m:46077

[Tr] S.R. TreIL, On superoptimal approximation by analytic and meromorphic matrix-valued functions, J. Functional Analysis 131 (1995), 386-414. MR 96g:47011

[V] N.P. VEKUA, Systems of singular integral equations and some boundary problems, GITTL, Moscow, 1950 (Russian); English transl., Noordhoff, Groningen, 1967. MR 13:247a

Department of Mathematics, Kansas State University, Manhattan, Kansas 66506

E-mail address: peller@math.ksu.edu 